\title{
Intravenous immunoglobulin treatment in paraneoplastic neurological syndromes with antineuronal autoantibodies
}

\author{
M Uchuya, F Graus, F Vega, R Reñé, J-Y Delattre
}

\begin{abstract}
Objective-To evaluate the effect of intravenous high dose human immunoglobulin (IVIg) therapy on the clinical course and autoantibody titres of patients with neurological paraneoplastic syndromes.

Methods-Twenty two patients with paraneoplastic encephalomyelitis and sensory neuronopathy syndrome associated with anti-Hu antibodies (18) or paraneoplastic cerebellar degeneration (PCD) with antiYo antibodies (four), were treated with 1-26 (mean 5.8) cycles of IVIg. The Rankin scale was used to evaluate the response.
\end{abstract}

Results-The only serious toxicity was one case of haemolytic anaemia. Twenty one patients were evaluable for therapeutic response. One patient, with subacute sensory neuronopathy (SSN), improved for at least 15 months, 10 remained stable (eight with anti-Hu and two with anti-Yo antibodies), and 10 deteriorated (eight with anti-Hu and two with anti-Yo antibodies). In seven of the 10 patients who stabilised, the syndrome had already made a plateau when the treatment was started but three patients (one with anti-Hu and two with anti-Yo antibodies) who had still been progressing stabilised for six, eight, and more than 48 months, including one patient with SSN who achieved stabilisation when the neurological dysfunction was only moderate (Rankin scale = 3). Another patient with SSN and initial stable response worsened when IVIg was reduced and improved when it was increased. No significant predictive factors of outcome could be identified but improvement or stabilisation was more frequent in patients with isolated involvement of the peripheral nervous system (62\%) than in patients with evidence of CNS damage (37\%) at the onset of treatment. Stabilisation in patients with CNS involvement was only achieved when the neurological dysfunction was already severe (Rankin scale $>3$ ). The titres of autoantibodies did not change significantly.

Conclusion-Treatment with IVIg at the doses given in the present protocol was not effective in paraneoplastic CNS syndromes associated with antineuronal antibodies. The role of this regime in the treatment of SSN should be further evaluated.

$(\Im$ Neurol Neurosurg Psychiatry 1996;60:388-392)
Keywords: immunoglobulin treatment; paraneoplastic neurological syndromes; antineuronal autoantibodies; Rankin scale

Specific autoantibodies against neuronal antigens are found in serum and CSF of patients with paraneoplastic syndromes of the nervous system. The best characterised are the paraneoplastic encephalomyelitis/sensory neuronopathy syndrome with anti-Hu antibodies and small cell lung cancer ${ }^{12}$ and paraneoplastic cerebellar degeneration (PCD) with anti-Yo antibodies $^{34}$ and breast or ovarian cancer. Anti-Hu and anti-Yo antibodies are directed against antigens shared by the primary tumour and neurons suggesting that an autoimmune mechanism is responsible for the neurological syndrome. There is no recognised treatment. Treatment of the tumour is often not effective in arresting the progressive course of the disease and in many instances the tumour is not detected at the onset of paraneoplastic syndromes. To date, immunosuppressive treatments, including corticosteroids or plasmapheresis, have not been useful, with a few exceptions. ${ }^{5}$ In this study, we evaluated the effect of intravenous human immunoglobulin (IVIg), which has been a successful treatment in several autoimmune diseases. ${ }^{6-9}$

\section{Patients and methods}

Twenty two patients (nine women and 13 men) aged 35 to 78 (mean 59) years, with PCD with anti-Yo antibodies (four) or paraneoplastic encephalomyelitis/sensory neuronopathy syndrome with anti-Hu antibodies (18) received IVIg between 1990 and 1994 (table). Of 18 patients with anti-Hu antibodies, five had multifocal CNS involvement and 13 had no CNS involvement when the treatment began. Nine of the 13 patients had a subacute sensory neuronopathy (SSN) and four a sensorimotor neuropathy. In addition, seven of the 13 patients with neuropathy also presented signs of dysautonomia. Four patients had intestinal pseudo-obstruction, two orthostatic hypotension (one of them also had areflectic mydriasis), and one impotence. The four patients with PCD had an isolated pancerebellar syndrome. No patient started the IVIg treatment in the month after the onset of the neurological dysfunction and only four did so in the second month (table). The median delay between development of paraneoplastic symptoms and IVIg was five (range 2 to 24) months. The neurological syndrome was stable when IVIg treatment started in 11 patients 


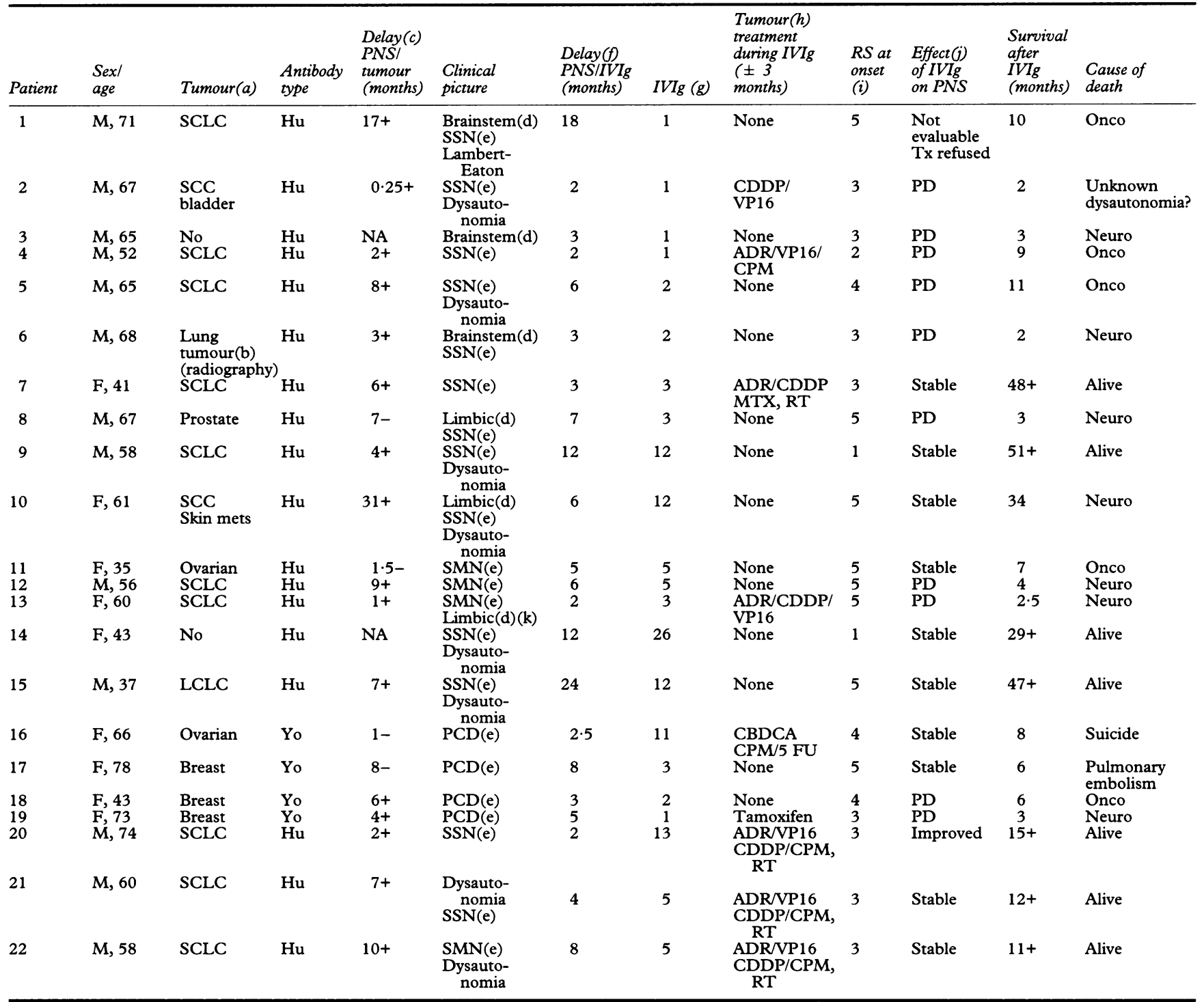

(a) Tumour: SCLC = small cell lung cancer; SCC = small cell cancer; LCLC = large cell lung cancer; No = no tumour found. (b) No histology. (c) Delay between the occurrence of the paraneoplastic neurological syndrome (PNS) and discovery of the tumour: + the PNS precedes the tumour; - the PNS follows the tumour; NA $=$ not appropriate. (d) Brainstem = brainstem encephalitis; limbic = limbic encephalitis. (e) SSN = subacute sensory neuronopathy; SMN = sensorimotor neuronopathy; PCD = paraneoplastic cerebellar degeneration. ( $f$ ) Delay PNS/IVIg = Delay between the occurrence of PNS and the onset of IVIg. (g) Number of IVIg cycles. (h) CDDP = cisplatin; ADR = adriamycin; VP16 = etoposide; MTX = methotrexate; CPM = cyclophosphamide; CBDCA = carboplatin; 5 FU = 5-fluorouracil; RT = radiotherapy. (i) Rankin scale at onset. (j) Effect of IVIg on paraneoplastic neurological syndrome. PD = progression. (k) Clinical evolution after IVIg courses.

$(50 \%)$. Stable disease was defined as the absence of progression of the neurological syndrome in the month before the onset of treatment.

\section{NATURE AND TREATMENT OF THE PRIMARY TUMOUR}

A tumour was found in 20 patients. In 16, the neurological syndrome preceded the tumour diagnosis (mean time $7 \cdot 3$ (range $0-31$ ) months). The neurological syndrome occurred after the tumour diagnosis (mean time 4.4 (range: 1-8) months) in four patients (two with PCD and two with paraneoplastic encephalomyelitis/sensory neuronopathy syndrome). In patients with anti-Hu antibodies, the tumour histology was small cell cancer (12 patients), large cell lung cancer (one), prostate adenocarcinoma (one), and ovarian dysgerminoma (one). Histological confirmation was not obtained in one patient with a mass lesion on chest radiography. No tumour was found in two patients with anti-Hu antibodies after a total follow up of three and more than 29 months. In patients with anti-Yo antibodies, the tumours found were breast (three) and ovarian (one). Seven patients received treatment for the tumour before IVIg. In three of them the paraneoplastic neurological syndromes appeared despite the tumour treatment. In the other four, they were known at the time of tumour treatment but none improved and only two stabilised. Nine patients had treatment of the tumour during IVIg therapy (table).

THERAPEUTIC PROTOCOL

Twenty two patients were treated with IVIg. Patients received IVIg, $0.5 \mathrm{~g} / \mathrm{kg} /$ day for five days. Treatment was repeated every four weeks for three months. IVIg was continued for six months or more in stable or improving patients $(0.5 \mathrm{~g} / \mathrm{kg} /$ day for one day, monthly). Neurological impairment was evaluated by a 
modified Rankin scale. ${ }^{510}(0=$ asymptomatic patient; 1 = non-disabling symptoms which do not interfere with lifestyle; $2=$ minor disabling symptoms, which lead to some restriction of lifestyle but do not prevent totally independent existence; 3 = symptoms significantly interfere with lifestyle or prevent totally independent existence; $4=$ moderately severe disabling symptoms which clearly prevent independent existence with total support for basic daily activities; $5=$ severe disabling, totally dependent requiring constant attention day and night; $6=$ death from neurological symptoms). Symptoms were considered improved or deteriorated if there was a change of at least 1 point in the Rankin scale. Stabilisation was defined as no change of Rankin scale after 3 courses of IVIg.

The detection of anti-Hu and anti-Yo antibodies was done by indirect immunoperoxidase on frozen sections of normal human brain cortex or cerebellum, and confirmed by western blot analysis. ${ }^{511}$ Serum and CSF anti-Hu and anti-Yo titres were defined as the reciprocal value of the highest dilution that demonstrated neuronal nuclear or Purkinje cell staining. Antibody titrations were done monthly before each treatment.

\section{Results}

TOLERANCE

Patients received 1-26 cycles of IVIg (mean: $5 \cdot 8$ ). Tolerance was good in 19 patients. Three patients had side effects of IVIg: one had transient headache at each cycle, one had a transient episode of fever, malaise, and cyanosis but the IVIg could be continued and one (patient 18, table) had a severe haemolytic anaemia requiring discontinuation of IVIg after two courses.

\section{THERAPEUTIC EFFECTS}

Twenty one patients were evaluable for therapeutic response. One patient $(1$, table) refused further treatment after one course. One patient $(20$, table) with a subacute sensory neuronopathy (SSN) improved. In this patient, who also received antitumour treatment, symptoms were progressing when the treatment began. He could not walk alone at the onset of treatment but after one course, he became able to walk easily and even to ride a bicycle and to go hunting. He remains improved 15 months later. Ten patients were considered stable (five SSN, two sensorimotor neuropathy, one paraneoplastic encephalomyelitis, two PCD). However, seven of them were already stable at the onset of IVIg. The three patients who were progressing $(17,16$, 7 , table) stabilised after the first course of IVIg and remained so for 6,8 , and more than 48 months with IVIg. Among them, two patients had PCD with severe cerebellar dysfunction (Rankin scale of 4 and 5) whereas the third patient with a SSN remained stable with a moderate deficit (Rankin scale of 3). An unusual course was observed in one patient $(14$, table) with a SSN who had stable sensory symptoms and a Rankin scale of 1 before initi- ation of therapy. She was stable during the first three cycles of IVIg and therefore fulfilled the criteria for stabilisation. However, she developed a dysautonomia (subocclusion) when the dose of IVIg was reduced to one treatment per month. A new cycle of five days treatment was restarted and the dysautonomia improved. A second deterioration with increased sensory ataxia occurred 10 months later after slow reduction of the IVIg doses. She improved again after an increased dosage. Twenty nine months after the onset of treatment she still had a Rankin scale of 1 . Finally, 10 patients deteriorated during IVIg (three with paraneoplastic encephalomyelitis, two with sensorimotor neuropathy, three with $\mathrm{SSN}$, and two with PCD). One of them (2, table), with SSN and severe orthostatic hypotension died suddenly from unknown reasons two months after the first cycle. He was considered a treatment failure despite a clear improvement of the orthostatic hypotension during this period because we could not rule out dysautonomia as the cause of death. ${ }^{2}$

We did not identify significant predictive factors of response or stabilisation including the delay in the onset of IVIg, the degree of disability before IVIg, or concomitant treatment of the tumour. However, $62 \%(8 / 13)$ of patients with symptoms restricted to the peripheral nervous system improved or stabilised whereas none of the patients with CNS involvement (paraneoplastic encephalomyelitis or PCD) improved and only $37.5 \%$ (3/8) of them stabilised. In all patients with CNS involvement stabilisation was achieved when there was already severe neurological dysfunction (Rankin scale $>3$ ). Median survival was six months for the entire group. Fourteen patients died, seven from neurological disease, four from tumour progression, one from pulmonary embolism, one from suicide, and one had a sudden unexplained death.

The serum titres of the antibodies did not change significantly during the IVIg treatment although there was a trend toward reduction of the titres in five evaluable patients $(17200$ (SD 13682) before, 11600 (SD 11696) after one course, 8400 (SD 4979) after two courses, 9200 (SD 4381) after three courses). Titres in CSF were not measured.

\section{Discussion}

Human immunoglobulins have been used with success in several neurological diseases with a presumed or proved autoimmune basis such as chronic inflammatory demyelinating polyneuropathy, ${ }^{71012}$ dermatomyositis, ${ }^{8}$ or myasthenia gravis. ${ }^{13}$

This is the first study to evaluate the effect of IVIg therapy in a series of patients with paraneoplastic neurological syndromes and antineuronal antibodies. The study was designed as an open preliminary trial looking for a clear positive result that had an impact on the degree of disability of the patients. Although we recognise that these trials are not the best to evaluate a given treatment, it is highly unlikely that a double blind randomised 
trial can ever be designed in these disorders due to their low frequency.

In the present study, improvement was only seen in one patient but stabilisation occurred in $10(47 \cdot 6 \%)$. The patient who improved also received concomitant antitumour treatment. It is therefore possible that antitumour treatment was in part responsible for neurological improvement. Although there are a few reports of regression of paraneoplastic neurological syndromes after treatment of the primary tumour alone ${ }^{14}$ and one report of spontaneous improvement, ${ }^{15}$ two reasons make us believe that IVIg therapy was of some benefit: (1) neurological improvement after treatment of the tumour is very rare in patients with paraneoplastic neurological syndromes and circulating autoantibodies. In a review of 71 patients with the $\mathrm{Hu}$ syndrome, Dalmau et $a l^{2}$ could not find a single case of improvement after treatment of the primary tumour. In addition we also found no case of improvement in a previous study of plasmapheresis and antineoplastic treatment in 16 patients with paraneoplastic neurological syndromes with antineuronal antibodies. ${ }^{5}$ (2) Four of our patients had already received antitumour treatment before IVIg at a time when they were neurologically symptomatic, without improvement of paraneoplastic neurological syndromes.

Interpretation of stabilisation is difficult. Paraneoplastic encephalomyelitis and PCD are characterised by neuronal loss and therefore a stabilisation of the disorder may be considered an effective response to the treatment. Of the 10 patients who experienced stabilisation, seven were already stable before IVIg treatment, suggesting that they had either an indolent form of the disease ${ }^{16}$ or spontaneous stabilisation unrelated to the treatment which occurs sometimes when the patients are bedridden. Nevertheless, in one of the three patients who were progressing when IVIg was started, the SSN has remained stable for more than four years.

Analysis of our data suggests that the type of neurological involvement at the onset of treatment may have an influence on the outcome. Indeed, improvement or stabilisation with a moderate (Rankin scale $\leqslant 3$ ) degree of dysfunction in the setting of neurological progression at the onset of IVIg therapy, was only seen in two patients with SSN. In addition, another patient with SSN apparently became "IVIg dependent", as she deteriorated at each reduction of the IVIg dose with a return to her previous condition after increased dosage. We previously showed that the development of CNS symptoms is associated with the occurrence of an intrathecal synthesis of antineuronal antibodies, which rarely exist in patients with pure SSN. ${ }^{17}$ Because the high molecular weight $(146 \mathrm{kDa})$ of IgG prevents entry into the CNS through the blood-brain barrier, it could be speculated that IgG could not reach the target in patients with CNS symptoms. However, patients with stiff man syndrome and antiglutamic acid decarboxylase antibodies improve after IVIg when the cause of the neurological deficit is thought to be a pathological involvement of the spinal cord interneurons. ${ }^{18}$

Improvement or useful stabilisation was found only in patients with a low Rankin scale of 1-3, at the start of treatment and none of the patients with a Rankin scale of 4-5 could return to a good quality of life. These data, coupled with those of our previous study with plasmapheresis ${ }^{5}$ underline the need to start treatment early in patients who are still ambulatory if the neurological disease is clearly progressing. It is unlikely that patients with severe neurological disability (Rankin scale of 4-5) benefit from IVIg or any immunosuppressor therapy unless they are treated very early. In the two case reports ${ }^{19} 20$ of PCD with severe disabilities that improved after IVIg (with treatment of the tumour in one case and plasmapheresis in the other), treatment was started within four weeks of the first symptoms of PCD.

The mechanism of action of IVIg in autoimmune diseases is unclear. ${ }^{6}$ We did not find a significant effect of the treatment on the titres of serum autoantibodies even though there was a trend to a progressive reduction of the titres after three courses of IVIg. This feature suggests that in paraneoplastic neurological disorders the action of IVIg is not mediated by anti-idiotypic antibodies ${ }^{91}$ or down regulation of antibody production. ${ }^{722}$ However, the lack of consistent reduction in the titre of autoantibodies in the present series does not necessarily explain the modest clinical results. Previous studies of IVIg treatment in myasthenia gravis and in the Lambert-Eaton myasthenic syndrome could not show a correlation between the clinical response and the titre of autoantibodies. ${ }^{23} 24$

In conclusion, IVIg therapy at the doses given in the present study seems not to be effective in patients with paraneoplastic neurological syndromes affecting the CNS. The modification of the clinical course in three of the nine patients with SSN (32\%) after IVIg therapy suggests that the treatment should be further evaluated in this subgroup of patients with paraneoplastic neurological syndromes.

This study was supported in part by Association pour la Recherche sur les Tumeurs Cérébrales (ARTC), Paris, France. We thank Dr E Schuller and the staff of his laboratory of neuroimmunology for their collaboration.

1 Anderson NE, Rosenblum MK, Graus F, Wiley RG, Posner JB. Autoantibodies in paraneoplastic syndromes associated with small-cell lung cancer. Neurology 1988; 38:1391-8.

2 Dalmau J, Graus F, Rosenblum MK, Posner JB. Anti-Huassociated paraneoplastic encephalomyelitis/sensory neu-
ropathy. A clinical study of 71 patients. Medicine 1992 ; ropathy. A

3 Chabriat H, Chen QM, Poisson M, Delattre JY. Dégénérescence cérébelleuse paranéoplasique. Rev Neurol (Paris) 1994;150:105-14.

4 Peterson K, Rosemblum MK, Kotanides H, Posner JB Paraneoplastic cerebellar degeneration. I. A clinica analysis of 55 anti-Yo antibody-positive patients Neurology 1992;42:1931-7.

5 Graus F, Vega F, Delattre JY, et al. Plasmapheresis and antineoplastic treatment in CNS paraneoplastic syndromes with antineuronal autoantibodies. Neurology 1992;42:536-40

6 Cook D, Dalakas M, Galdi A, Biondi D, Porter H. Highdose intravenous immunoglobulin in the treatment of demyelinating neuropathy associated with monoclonal gammopathy. Neurology 1990;40:212-4.

7 Faed JM, Day B, Pollock M, Taylor PK, Nukada H, 
Hammond-Tooke GD. High-dose intravenous human immunoglobulin in chronic inflammatory demyelinating polyneuropathy. Neurology 1989;39:422-5.

8 Roifman CM, Schaffer FM, Wachsmuth SE, Murphy G, Gelfand EW. Reversal of chronic polymyositis following intravenous immune serum globulin therapy. $¥ A M A$ 1987;258:513-5.

9 Vermeulen M, Van der Meché FGA, Speelman JD, Weber A, Busch HFM. Plasma and gamma-globulin infusion in chronic inflammatory polyneuropathy. $\mathcal{f}$ Neurol Sci 1985;70:317-26.

10 Van Doorn PA, Brand A, Strengers PFW, Meulstee J, Vermeulen M. High-dose intravenous immunoglobulin treatment in chronic inflammatory demyelinating neutreatment in chronic inflammatory demyelinating neuropathy: a double-blind, placebo-cc
study. Neurology 1990;40:209-12.

11 Dalmau J, Posner JB. Neurologic paraneoplastic antibodies (anti-Yo; anti-Hu; anti-Ri): the case for a nomenclature based on antibody and antigen specificity. Neurology 1994;44:2241-6.

12 Vermeulen M, Van Doorn PA, Brand A, Strengers PFW, Jennekens FGI, Busch HFM. Intravenous immunoglobulin treatment in patients with chronic inflammatory demyelinating polyneuropathy: a double blind, placebo controlled study. $\mathcal{F}$ Neurol Neurosurg Psychiatry 1993; 56:36-9.

13 Soueidan SA, Dalakas MC. Treatment of autoimmune neuromuscular diseases with high-dose intravenous neuromuscular diseases with high-dose intravenous
immune globulin. Pediatr Res 1993;33(suppl):S95-S100.

14 Delattre JY, Davila L, Vega F, Poisson M. Auto-Immunité et syndromes neurologiques paranéoplasiques. Rev

15 Twijnstra A, Verschuuren J, Byrne TN, De Baets M, Posner J, Dalmau J. Prolonged or remitting Anti-Huassociated paraneoplastic sensory neuronopathy (PSN) and encephalomyelitis (PEM). Neurology 1995;45 (suppl 4):A321.

16 Graus F, Bonaventura I, Uchuya $M$, et al. Indolent antiHu-associated paraneoplastic sensory neuropathy. Neurology 1994;44:2258-61

17 Vega F, Graus F, Chen QM, Poisson M, Schuller E, Delattre JY. Intrathecal synthesis of the anti-Hu antibody in patients with paraneoplastic encephalomyelitis or sensory neuronopathy: clinical-immunologic correlation. Neurology 1994;44:2145-7.

18 Amato AA, Cornman EW, Kissel JT. Treatment of stiffman syndrome with intravenous immunoglobulin. man syndrome with int

19 Counsell CE, McLeod M, Grant R. Reversal of subacute paraneoplastic cerebellar syndrome with intravenous paraneoplastic cerebellar syndrome with

20 Moll JWB, Henzen-Logmans SC, Van der Meche FGA, Vecht $\mathrm{Ch}$ J. Early diagnosis and intravenous immune globulin therapy in paraneoplastic cerebellar degeneration. I Neurol Neurosurg Psychiatry 1993;56:112-5.

21 Dietrich G, Kaveri SV, Kazatchkine MD. Modulation of autoimmunity by intravenous immune globulin through interaction with the function of the immune/idiotypic network. Clin Immunol Immunopathol 1992;62:S73-S81.

22 Cornblath DR, Chaudry V, Griffin JW. Treatment of chronic inflammatory demyelinating polyneuropathy with intravenous immunoglobulin. Ann Neurol 1991;30. $104-6$

23 Gajdos P. Intravenous immune globulin in myasthenia gravis. Clin Exp Immunol 1994;97(suppl 1):49-51.

24 Takano $H$, Tanaka $M$, Koike $R$, Nagai $H$, Arakawa $M$, Tsuij S. Effect of intravenous immunoglobulin in Lambert-Eaton myasthenic syndrome with small-cell Lambert-Eaton myasthenic syndrome with small-cell calcium channel antibody. Muscle Nerve 1994;17:1073-5.

\section{NEUROLOGY IN LITERATURE}

\section{Doctors' remedies}

As the last of these extracts was written over 70 years ago, it might seem that their message has dimmed; far from it. Medicine (and surgery) can still be applied inappropriately, prolong life unreasonably, and create new disorders to replace those for which it was first used.

Fonathan Swift, 1726, Gulliver's travels

For nature (as the physicians alledge) having intended the superior anterior orifice only for the intromission of solids and liquids, and the inferior posterior for ejection; these artists ingeniously considering that in all diseases nature is forced out of her seat; therefore to replace her in it, the body must be treated in a manner directly contrary, by interchanging the use of each orifice; forcing solids and liquids in at the anus, and making evacuations at the mouth.

Laurence Sterne, 1759-67, The life and opinions of Tristram Shandy, gentleman

The stroke at the Prince of Physicians, with which he began, was no more than a short insult upon his sorrowful complaint of the ars longa,-and vita brevislife short, cried my father, - and the art of healing tedious! And who are we to thank for both, the one and the other, but the ignorance of quacks themselves, and the stage-loads of chymical nostrums, and peripatetic lumber, with which in all ages, they have first flattered the world, and at last deceived it.

\section{Mark Twain, 1894, Pudd'nhead Wilson}

The doctor asked for a few sheets of paper and a pen, and said he would write a prescription; which he did. It was one of Galen's; in fact, it was Galen's favorite, and had been slaying people for sixteen thousand years. Galen used it for everything, applied it to everything, said it would remove everything, from warts all the way through to lungs-and it generally did. Galen was still the only medical authority recognised in Missouri; his practice was the only practice known to the Missouri doctors, and his prescriptions were the only ammunition they carried when they went out for game. By and by Dr Claypool laid down his pen and read the result of his labors aloud, carefully and deliberately, for this batten must be constructed on the premises by the family, and mistakes could occur; for he wrote a doctor's hand-the hand which from the beginning of time has been so disastrous to the apothecary and so profitable to the undertaker ... "There," he said, "That will fix the patient; give his brother a dipperful every threequarters of an hour-" - "While he survives," muttered Luigi- . . .

During Monday, Tuesday, and Wednesday the twins grew steadily worse; but then the doctor was summoned south to attend his mother's funeral, and they got well in forty-eight hours.

\section{H G Wels, 1900, Tono Bungay}

Close at hand was the doctor with one of those cruel and idiotic injection needles modern science puts in the hands of these half-educated young men, keeping my uncle flickeringly alive for no reason whatever.

Marcel Proust, 1923, Remembrance of things past The captive

Nature scarcely seems capable of giving us any but quite short illnesses. But medicine has developed the art of prolonging them. Remedies, the respite that they procure, the relapses that a temporary cessation of them provokes, produce a simulacrum of illness to which the patient grows so accustomed that he ends by making it permanent, just as children have regular fits of coughing long after they have been cured of the whooping cough. Then the remedies begin to have less effect, the doses are increased, they cease to do any good, but they have begun to do harm thanks to this lasting indisposition. Nature would not have offered them so long a tenure. It is a great wonder that medicine can almost rival nature in forcing man to remain in bed to continue taking some drug on pain of death. From then on, the artificially grafted illness has taken root, has become a secondary but a genuine illness, with this difference only, that natural illnesses are cured, but never those which medicine creates, for it does not know the secret of their cure.

G D PERKIN Charing Cross Hospital, Fulham Palace Road, London W6 8RF, UK 